\title{
Efecto de la intervención nutricional multidisciplinaria ambulatoria en el peso de los pacientes ${ }^{1}$
}

Institución: Hospital Dr. Rafael Ángel Calderón Guardia

\section{COMO CITAR}

\section{RESUMEN}

Este artículo presenta los resultados de la intervención nutricional multidisciplinaria ambulatoria que se brinda en el Hospital Rafael Ángel Calderón Guardia. Esta consulta evita reingresos e infecciones y se educa al paciente y a la familia. La suplementación y los planes nutricionales dados por el grupo multidisciplinario reflejan el logro de aumento de peso del $74 \%$ de la muestra en estudio. La investigación es de carácter descriptivo-retrospectivo y está basada en los datos estadísticos generados en la consulta externa de soporte nutricional del Hospital Dr. Rafael Ángel Calderón Guardia. La población estuvo conformada por 100 pacientes que contaban con, al menos, 2 valoraciones en el 2011. El médico especialista y una enfermera capacitada en soporte nutricional conformaron el equipo que completó la muestra. Se analizó el impacto de las intervenciones nutricionales en el peso corporal de los atendidos; en cada cita, se registró el peso en kilogramos (Kg.), talla en metros, índice de masa corporal $\left(\mathrm{Kg} . / \mathrm{m}^{2}\right)$ y la ganancia de peso en kilogramos. Esta información se documentó mediante una hoja electrónica diseñada en los programas Word 2003 y Excel 2003. Las variables fueron: edad, sexo y diagnóstico. Con respecto a la evolución de los pacientes atendidos en la consulta externa de soporte nutricional, se encontró que 74 pacientes aumentaron de peso, lo cual representa un 74\%; 4 individuos (4\%) lograron mantener el peso registrado en la primera cita y 22 pacientes (22\%) continuaron perdiendo peso. Un abordaje multidisciplinario del soporte nutricional permite que la mayoría de los pacientes que, por su patología, presentan desnutrición o riesgo de padecerla, puedan mejorar o mantener su estado nutricional.

Palabras clave: Grupo multidisciplinario- intervención - peso - suplementación.

\footnotetext{
${ }^{1}$ Fecha de recepción: 10 de mayo del 2012

Fecha de aceptación: 19 setiembre del 2012

${ }^{2}$ Enfermera. Especialista en Nutrición Enteral del Hospital Rafael Angel Calderón Guardia. Correo electrónico: marialuisa.fallas@gmail.com
} 


\title{
Effect of nutritional intervention on weight multidisciplinary ambulatory patients ${ }^{1}$
}

Institution: Hospital Dr. Rafael Ángel Calderón Guardia

\section{CITED}

\begin{abstract}
This retrospective descriptive study is based on statistical data generated in the outpatient nutritional support of Dr. RA Calderon Guardia Hospital. Educate the patient and their family. Further, the nutritional plans and nutritional supplementation given by the multidisciplinary group shows a weight gain of $74 \%$ of the study group. The sample population was 156 patients who presented during calendar 2011. The population consisted of 100 patients who had at least 2 evaluations in 2011. The analysis evaluated the impact of nutritional interventions on body weight of those treated. At each patient examination, weight was measured in kilograms $(\mathrm{kg})$, height in meters, body mass index as $(\mathrm{kg} / \mathrm{m} 2)$, and changes in weight in kilograms. The spreadsheet designed and used for data and graphs was Word 2003 and Excel 2003. The doctor and a nurse specialist trained in nutritional support, the team was complete the sample. Variables were: Age, Sex, Patient Diagnosis Regarding the outcome of patients treated in the outpatient, nutritional support found that 74 patients gained weight which represents $74 \%$ of the sample group, 4 patients $(4 \%)$ were able to maintain weight, and 22 patients $(22 \%)$ continued to lose weight. A multidisciplinary approach to nutritional support allows most of patients by their pathology or malnutrition to submit increased risk of suffering, can improve or maintain their nutritional status
\end{abstract}

Key word: multidisciplinary group-intervention - weight - supplementation.

\footnotetext{
${ }^{1}$ Date of reception: May 10, 2012

Date of acceptance: september 19, 2012

${ }^{2}$ Nurse. Enteral Nutrition Specialist, Rafael Angel Calderón Guardia Hospital. E-mail: marialuisa.fallas@gmail.com
} 


\section{INTRODUCCIÓN}

Las tendencias actuales en el campo de la salud involucran el trabajo multidisciplinario como parte de las estrategias para mejorar la calidad de vida de las personas.

El soporte nutricional no es la excepción, ya que la malnutrición, más frecuente en los pacientes con enfermedades graves, tiene efectos importantes en la salud de ellos; además, es importante reconocer el gran costo de esta.

Gómez, Cantón, Luengo y Olviera (2010) afirman que: "La malnutrición proteica -calórica, puede afectar 30 o 50\% de los pacientes hospitalizados, aumenta el tiempo de hospitalización y el costo de atención médica en aquellos que la padecen.” (p. 781).

En Costa Rica, el manejo de la malnutrición, desde todas sus aristas, es muy reciente e incluye el uso de macro y micronutrientes. Estudio latinoamericano multicéntrico indicó que "el 48,1\% de pacientes hospitalizados están desnutridos y un 12,6\% presentan mal nutrición severa.

"Brasil y Costa Rica son los únicos países que cuentan con políticas gubernamentales, con equipos de terapia nutricional” (María Isabel Correia (2003, p.427).

Dicho estudio los datos de desnutrición en Costa Rica fue de desnutrición moderada 50.3\% y severa $18.1 \%$ (ELAN, 2003).

\section{Según Gómez et al.(2010),}

"Un importante volumen de evidencias científicas avala que la administración de suplementos nutricionales mejora el estado nutricional o previene la aparición de malnutrición en pacientes que no cubren sus necesidades de ingesta. (p.781).

El propósito de este estudio es evaluar el efecto de la Intervención nutricional multidisciplinaria ambulatoria en el peso de los pacientes en el año 2011.

El equipo multidisciplinario de soporte nutricional se creó en el año 1991 con la finalidad de brindar atención a pacientes hospitalizados que requirieran de nutrición enteral o parenteral durante su internamiento. El equipo consta de una enfermera, una nutricionista, una farmacéutica y un médico que funge como coordinador.

Con el paso del tiempo, el equipo detectó la necesidad de ampliar su cobertura a los pacientes ambulatorios que sufrían de patologías como cáncer, neuropatías, parálisis cerebral infantil, enfermedades digestivas, demencias; además, se incluyó a pacientes adultos mayores desnutridos. Lo anterior con el objetivo de lograr un resultado satisfactorio que se reflejara en el mejoramiento o en el mantenimiento del estado nutricional 
en estos pacientes; la bibliografía y los expertos en el tema indican que la atención debe ser brindada por un equipo multidisciplinario.

Sin embargo, por deficiencia de recursos humanos, no había sido posible iniciar la consulta externa, sino hasta octubre de 2009; esto gracias al esfuerzo de los miembros del equipo.

\section{MATERIALES Y METODO}

Se realizó un estudio descriptivo- retrospectivo basado en las estadísticas generadas en la consulta externa de soporte nutricional del Hospital Dr. Rafael Ángel Calderón Guardia. La población fue de 156 personas atendidas durante el año 2011.

El criterio de inclusión para la evaluación del comportamiento según el peso fue contar con, al menos, dos registros antropométricos durante el año en estudio; 56 pacientes fueron excluidos, pues únicamente contaban con un registro antropométrico. Lo anterior pudo deberse a diferentes motivos: el paciente asistió en una sola oportunidad, por su condición tuvo consultas indirectas o que, por alguna razón, su información no apareciera en el registro antropométrico de las estadísticas.

Por lo tanto, la muestra de la población estuvo conformada por 100 pacientes con al menos dos valoraciones en el año 2011. Todos los enfermos contaban con una evaluación médica, además de medidas antropométricas, análisis bioquímico y dietético, cálculo de requerimientos y plan de alimentación o recomendaciones nutricionales, dependiendo del caso. También, los pacientes recibieron suplementación y medicación de acuerdo con sus necesidades.

\section{Las medidas antropométricas fueron las siguientes:}

Toma de peso y estatura: en pacientes con capacidad para movilizarse, se utilizó la balanza de plataforma con tallímetro.

Para determinar el peso, se debe tomar en cuenta los siguientes aspectos:

a) La balanza debe ser calibrada.

b) El paciente debe estar libre de cualquier objeto pesado (llaves, billeteras, monedas entre otros).

c) El peso se debe tomar sin zapatos.

Para registrar la talla, es necesario asegurarse de que la posición del paciente sea la indicada: pies juntos y cabeza erguida. 
.

\section{Revista Electrónica Enfermeria Actual en costa Rica}

\section{www.revenf.ucr.ac.cr}

La toma de peso y talla la realiza el profesional de Nutrición o de Enfermería entrenado para llevar a cabo dicha tarea.

Cuando el paciente se encuentra encamado o es incapaz de movilizarse, se debe estimar el peso y la talla mediante otras medidas corporales, tales como la altura de rodilla y la circunferencia braquial. En estos casos, se aplican fórmulas matemáticas que toman en cuenta edad, género, tallas y mediciones aproximadas para estimar el peso y la talla del paciente. El proceso lo lleva a cabo un profesional, previamente entrenado, con el uso de una cinta métrica calibrada.

Para la realización de este estudio se contó con el consentimiento del Comité Bioético del Hospital Dr. Rafael Angel Calderón Guardia.

En cada cita, se registró el peso en Kilogramos (Kg.), talla en metros, índice de masa corporal, $\left(\mathrm{Kg} . / \mathrm{m}^{2}\right)$ y ganancia de peso en kilogramos; esto se documentó mediante una hoja electrónica diseñada para este estudio. investigador

El médico especialista y una enfermera capacitada en soporte nutricional conformaron el equipo

Las variables fueron:

- edad

- sexo

- diagnóstico

- lugar de procedencia

\section{RESULTADOS}

La cantidad de pacientes valorados en la consulta multidisciplinaria de soporte nutricional ha incrementado con el paso de los años.

Los pacientes que consultaron, durante este último año, fueron atendidos por un equipo multidisciplinario conformado por una enfermera, un médico y una nutricionista.

La distribución de los pacientes, según género, fue la siguiente: 57 mujeres y 43 hombres. Respecto de la edad, los usuarios atendidos oscilaron entre los 13 y los 99 años. Existe un número importante de individuos entre los 13 y los 23 años, quienes en su mayoría padecen de parálisis cerebral infantil y fueron referidos desde el Hospital Nacional de Niños para su seguimiento. 
En cuanto al progreso y evolución de los atendidos en la consulta de soporte nutricional, se encontró que 74 de ellos aumentaron de peso, cuatro mantuvieron el peso registrado en la primera cita y 22, continuaron perdiendo peso.

$\mathrm{Al}$ analizar los datos anteriores en relación con el sexo, se determinó que, en el caso de las mujeres, el $73.6 \%$ logró aumentar de peso, mientras que esto sucedió solo en el $65 \%$ de los hombres. Con respecto a quienes lograron mantener el peso, la población femenina representa un $5.3 \%$ y la masculina, un $2.3 \%$. Con respecto a los pacientes que perdieron peso a pesar de la intervención el $21 \%$ fueron mujeres y el $32 \%$, hombres.

\section{DISCUSIÓN}

En el año 2009, se inició la consulta en el mes de octubre por lo que la cantidad de pacientes valorada fue solo de nueve; sin embargo, esta incrementó considerablemente. En el 2010, alcanzó los 136 pacientes nuevos y, en el 2011, los 156 .

Es importante mencionar que, al analizar los datos presentados en el apartado anterior, se determinó que el $31 \%$ de los pacientes atendidos en la consulta multidisciplinaria de soporte nutricional padece de alguna enfermedad oncológica, predominantemente de cabeza y cuello o de tracto gastrointestinal. Otro porcentaje importante (22\%) lo ocupan las neumopatías -EPOC, fibrosis quística y fibrosis intersticial difusa-; estos pacientes forman parte del Programa de Rehabilitación Pulmonar.

El abordaje de los pacientes con patologías como las mencionadas tanto en el prequirúrgico, con el fin de mejorar el estado nutricional para someterse a diversas cirugías, así como en el post quirúrgico, por resecciones importantes a nivel de tracto gastrointestinal, representa el $7 \%$ de los abordajes de soporte nutricional.

Es importante rescatar estos datos, ya que repercuten directamente sobre el estado nutricional de las personas atendidas y por el hecho de que se requiere de una intervención multidisciplinaria para su abordaje.

En este sentido, Arenas y Anaya (2007) mencionan que, para los pacientes con cáncer, la mejor terapia nutricional es la que resulta más fisiológica e implica menos complicaciones; la vía oral debe ser siempre la primera opción considerada. Con base en esa premisa, el uso de suplementos y consejo dietético debe contemplarse como el primer recurso para mejorar el estado nutricional del portador de alguna patología oncológica. En el paciente con cáncer, la terapia nutricional puede mejorar la calidad de vida aún en quimioterapia y radioterapia.

Actualmente, debido a las características propias de estos enfermos, se recomienda las dietas poliméricas, de alta densidad calórica, hiperproteicas, con fibra o sin ella, las cuales deben mantenerse hasta que la ingesta oral sea la óptima (Gil, 2010, p.561). 
Además de los pacientes con cáncer, otro grupo que se ve beneficiado por el programa son los individuos que presentan diversas enfermedades neurológicas, como demencias y eventos cerebro vasculares; estos representan el $13 \%$ de los pacientes atendidos.

Así como el grupo mencionado; los avances tecnológicos médicos han permitido que las personas con secuelas de parálisis cerebral infantil (PCI) que alcanzan la edad adulta sean cada vez mayores. La gran mayoría de esta población requiere de un abordaje nutricional multidisciplinario; se destaca que representan el $9 \%$ del total de pacientes atendidos.

Estos pacientes necesitan de un soporte nutricional multidisciplinario, ya que la enfermedad neurológica que padecen les ocasiona disfagia y otras sintomatologías que les impiden alimentarse de forma tradicional. En gran proporción, también precisan de la colocación de sondas y de ajustes especiales en su alimentación.

Arenas y Anaya (2007) explican que los pacientes con daño neurológico frecuentemente sufren de una importante dificultad para alimentarse por vía oral y con alimentos naturales. Esto se debe a la afectación de las funciones cerebrales superiores, las alteraciones de la sensopercepción del ambiente, las discapacidades motoras de la deglución y coordinación y el deterioro del estado de alerta, así como de la respuesta metabólica endocrina al traumatismo que se expresa con gran hiper metabolismo e hipercatabolia. Todo lo anterior lleva a un deterioro temprano de la composición corporal, lo cual afecta el compartimento graso, pero, sobre todo, la masa magra. Esto aumenta la frecuencia de infecciones nosocomiales, la duración de la estancia hospitalaria y el número de muertes.

En estos casos, resulta de suma importancia que la intervención nutricional sea intensa, ordenada y que, en la medida de lo posible, tanto el cuidador como el paciente formen parte de esta para que ambos asuman la terapia nutricional como un aspecto fundamental del proceso de recuperación.

Otras situaciones de salud como el virus de la inmunodeficiencia humana (VIH), preparación para trasplante hepático, manejo posterior a este y a trasplantes cardíacos, así como el Síndrome de inmovilización y enfermedades reumatológicas, ocupa un $18 \%$. Lo anterior tiene repercusión en la nutrición del individuo enfermo ya que, la desnutrición incrementa la vulnerabilidad de las personas a contraer enfermedades y condiciona la evolución y el pronóstico de la enfermedad (Sánchez, Díaz, Verga, Sánchez, Alfonso, 2009, p. 113).

Con la información presentada, se evidencia el impacto que el equipo de soporte nutricional del HCG tiene en un sinnúmero de patologías, ya que, como se explicó, después de la intervención se logra una considerable ganancia de peso en los usuarios del programa.

Además, Costa Rica posee un sistema de salud de alta calidad y los servicios hospitalarios han evolucionado significativamente. También, ha alcanzado gran complejidad hospitalaria en cada servicio de los 
nosocomios; esto ha repercutido en la necesidad de creación de equipos de apoyo, en las diversas áreas, para garantizar la adecuada recuperación de los pacientes.

El Soporte Nutricional es uno de los sectores que brinda apoyo para garantizar la evolución adecuada de los pacientes hospitalizados; por esto ha logrado, con la apertura del programa ambulatorio, un aumento significativo del número de pacientes captados. Tal y como se presentó en el apartado anterior, los resultados han sido positivos; esto se debió en gran medida, al trabajo en equipo.

Dentro de la historia del paciente, también se reporto el estado socioeconómico y finalmente, se documentó el nivel educativo de los cuidadores y/o familiares en el manejo de la sonda de alimentación (si la tuviese) y en el almacenaje de material y equipo.

Según Arenas (2007):

Las ventajas de un equipo nutricional es que permite una indicación precisa, el uso racional de técnicas, la ampliación de la cobertura, la puesta en práctica de soporte nutricional en el hogar y la optimización de recursos, controlando el costo y manteniendo la calidad. Además agrega que el funcionamiento adecuado exige una organización óptima basada en normas y procedimientos escritos. (p. 556).

Por lo expuesto, en la consulta ambulatoria de soporte nutricional se cuenta con protocolos que incluyen una evaluación médica y nutricional (antropométrica y dietética), así como una asesoría, desde la perspectiva de Enfermería, con respecto al manejo de las sondas (según normas establecidas) H.C.G.(C.C.S.S).

En cuanto a la distribución por edades, la mayoría de los pacientes valorados en la consulta ambulatoria de soporte nutricional se encuentran entre los 57 y los 87 años. Esto último coincide con el rango de edades en el que más frecuentemente se presentan las patologías que ameritan de este tipo de intervención. Para el cáncer gástrico, por ejemplo, el pico máximo de edad se encuentra entre los 50 y 70 años con una incidencia máxima entre los 60-65 años. "La sobrevivencia de los pacientes con cáncer gástrico es muy baja debido a que en la mayoría de los casos se detectan a edad avanzada" (Fallas 2009, p. 427) . Con respecto al "EPOC en la población geriátrica existen cifras elevadas de esta patología, debido a que su diagnostico en la consulta de atención primaria de salud no es inicial, sino que en su mayoría el porcentaje es de control y seguimiento, es decir de tratamiento crónico." (Guzmán 2008, p. 244).

En cuanto a esta población la edad, en la mayoría de ellas la muerte ocurre a los 65 años (Guzmán 2008, p.245)

Cabe rescatar también, que el equipo de soporte nutricional evalúa y brinda tratamiento a los pacientes geriátricos con bajo peso, lo que aumenta la población con las edades descritas. 
Los pacientes atendidos en la consulta externa recibieron consejo nutricional individualizado, el cual fue evaluado y readaptado en cada una de la consultas pues, como bien lo demuestra la literatura,

Un tratamiento nutricional intensivo e individualizado contribuye a mejorar la calidad de vida de los pacientes, si es aplicado en el estadio temprano de la enfermedad. Además, se agrega que gran parte de los pacientes oncológicos se puede beneficiar de recomendaciones dietéticas generales y específicas, para el control de su enfermedad o para el tratamiento relacionado con el control de los síntomas, lo que ayuda a mantener un adecuado estado nutricional y prevenir la malnutrición. (Marín, Liviano, Pichard y Gómez. 2007, p.337)

A los pacientes evaluados se les indicó una fórmula enteral completa como suplemento nutricional. Según Marín et al (2007), dicha

Suplementación oral en pacientes portadores de enfermedades crónicas que impactan el estado nutricional ha mostrado beneficios en términos de funcionalidad física y de ganancia de peso. Afirman, además, que en pacientes con un Índice de Masa Corporal (IMC) $<20 \mathrm{~kg} / \mathrm{m}^{2}$ los beneficios han sido más pronunciados (Marín et al 2007, p.458).

Otros autores sostienen que los suplementos nutricionales, mas allá de su eficacia, constituyen una terapéutica segura sin efectos adversos clínicos relevantes, lo cual de acuerdo con la literatura existente, mejora tanto la funcionalidad del paciente como su calidad de vida.

Por otro lado, las enfermedades pulmonares también impactan en el estado nutricional, tal y como lo indican Fuchs y Sandoval (2005) que "las neumopatías suelen afectar de manera adversa la ingestión alimentaria, de tal manera que los enfermos están expuestos al peligro de la desnutrición” (p. 5).

Algunas neumopatías “incrementan de manera sustancial las necesidades energéticas y proteicas, pero las complicaciones de la enfermedad o su tratamiento pueden dificultar la ingestión y la retención adecuada de nutrientes" (Fuchs, Sandoval, 2005, p.5)

Lo anterior se aplica a la práctica en el Hospital Calderón Guardia donde los pacientes que presentan algún tipo de neumopatía son referidos por el Servicio de Rehabilitación Pulmonar, donde realizan ejercicios físicos y pulmonares bajo la supervisión de un licenciado en Terapia respiratoria. Este último los refiere a la consulta de soporte nutricional con el objetivo de mejorar su estado de salud y así aumentar la expansión pulmonar. El tratamiento del EPOC pretende incrementar la tolerancia al ejercicio, mejorar el estado de salud, disminuir los síntomas y prevenir las complicaciones, así como las exacerbaciones. Ya que, "La pérdida de peso y 
especialmente de masa muscular contribuye de modo considerable a la morbilidad, incapacidad y desventaja en pacientes con EPOC”. (Arenas y Anaya, 2007, p.350).

La desnutrición que habitualmente acompaña al EPOC es un factor predictivo independiente de una alta morbi-mortalidad, una menor calidad de vida y de una menor capacidad aeróbica. La desnutrición proteica genera cambios intrínsecos en el metabolismo muscular, lo cual disminuye la fuerza muscular tanto general como respiratoria; esto no siempre se refleja en las habituales herramientas de evaluación nutricional (Consenso Argentino de Rehabilitación Respiratoria, 2004).

Por otro lado, Gil, A. (2010) señala que la vigilancia de la ingesta alimentaria del paciente con EPOC es clave de cara a la evolución y pronóstico de la enfermedad. (p.575).

Actualmente, resulta cada vez más frecuente tomar en cuenta el estado nutricional, como un aspecto fundamental, antes de someter al paciente a una cirugía de tipo electiva. En individuos con desnutrición moderada o severa, sobre todo, se considera importante una repleción que mejore los resultados de la cirugía. Lo anterior debido a que la cirugía, en sí, implica ayuno de tiempo variable, estrés quirúrgico y aumento de la tasa metabólica. Estos problemas son mayores si la cirugía es en el tubo digestivo. (Arenas y Anaya, 2007, p.455).

Respecto de la cita anterior, Gil, A. (2010) dice que

La ruta óptima para la nutrición preoperatoria es la enteral, oral o por sonda; la evidencia actual demuestra que el mayor beneficio se obtiene en aquellos pacientes con desnutrición grave, independientemente de su etiología. Este soporte debe darse por al menos 7 días para que sea efectivo. (p. 536).

El equipo multidisciplinario brinda un excelente servicio, en atención, educación y suplementación, al paciente de consulta externa. La enfermera juega un papel relevante en el grupo multidisciplinario, pues indaga acerca de las condiciones socio-económicas de los pacientes; además, educa y coordina con las redes del área de atracción para dar material y equipo. También, forma parte de los procedimientos, por ejemplo, asiste al médico en los cambios de las sondas de alimentación (P.E.G.-Gastrostomias) y realiza las medidas antropométricas en conjunto con la nutricionista. Además, brinda seguimiento y control por teléfono; en casos de mal funcionamiento de la sonda de alimentación (rupturas de Y, balón desinflado), guía, por vía telefónica, al cuidador o familiares para la corrección del desperfecto mecánico de la sonda; esto para evitar trasladar al paciente a la consulta externa. Participa en la recolección de datos estadísticos de los pacientes que asisten a la consulta y, finalmente, forma parte de los equipos de enfermeras (os) del alta hospitalaria en actualización con las redes de atención primaria. 


\section{Revista Electrónica Enfermería Actual en costa Rica}

Los pacientes, frecuentemente, expresan satisfacción en cuanto a la atención brindada y los resultados obtenidos.

\section{CONCLUSIÓN}

Un abordaje multidisciplinario de soporte nutricional permite que la mayoría de los pacientes, quienes por su patología presentan desnutrición o alto riesgo de padecerla, puedan mejorar o mantener su estado nutricional.

El suplemento, por su facilidad de preparación y alto aporte calórico y proteico, es de gran ayuda para que los pacientes mejoren o mantengan su estado nutricional.

\section{AGRADECIMIENTO}

Doy gracias a la Coordinadora de Soporte Nutricional del Hospital Dr. Rafael Ángel Calderón Guardia, la Dra. Paola Sánchez Corrales, médico general y nutrición, y a la nutricionista del mismo centro, la Dra. Cecilia Vargas Hernández, por su aporte bibliográfico y estadístico en el trabajo de estudio.

\section{REFERENCIAS}

Arenas. H, y Anaya. R, (2007). Nutrición Enteral y Parenteral. México D.F.: Editorial Mc. Graw Hill. (p. 600).

Correia M, Antonio CL, (2003) Prevalencia de Desnutrición Hospitalaria en Latinoamérica, Estudio Multi Céntrico ELAN, Revista Médica Panamericana. 28 (10) 823-825.

Fallas. M, (2009) Efecto de la Erradicación Helicobacter Pylori, en la Prevención de Cáncer Gástrico. Revista Médica de Costa Rica y Centro America, 16 (590) 427- 429

Fush, V. y Sandoval, J. (2005) Soporte Nutricional en el Paciente Neumópata. Revista de Neumología y Cirugía de Tórax, 64 (1) 5-8

Gómez. C, Cantón. C, Pérez. L, Olveira. M, Fuster. G. (2010) Eficacia, Costo- Efectividad y Efectos Sobre la Calidad de Vida de la Suplementación Nutricional. Nutrición Hospitalaria, 25 (5) 781-792

Gil, A. (2010). Tratado de Nutrición, Desnutrición y Enfermedad. Nutrición Clínica. Tomo 4. Madrid: Editorial Panamericana.

Guzmán. R, (Octubre 2008) EPOC en el Adulto Mayor, Control de Síntomas en Consulta Médica de Familia. Rev. Clin. Fam. 23 (5) $244-249$

Marín, M.M., Gómez, C., Castillo, R., Lourenço, T., García, M., Loria, V., Villarino, M., Zamora, P., Luengo, L., Robledo, P., López-Portabella' C., Zarazaga, A., Espinosa, J., Nogués, R., Rodríguez, L., Celaya, S. y Pardo J. (2008) Evaluación del Riesgo Nutricional e Instauración de Soporte Nutricional en Pacientes Oncológicos, Nutr.Hosp. 23(5) 458 468

Marín, M., Laviano, A., Pichard, C. y Gómez, C. (Mayo-Junio, 2007) Relación entre la intervención nutricional y la calidad de vida en el paciente con cáncer. Nutrición Hospitalaria. Nutr. Hosp. 22 (3) 337-350 


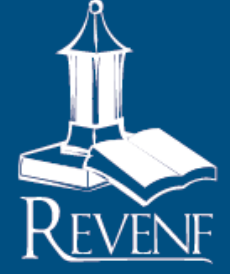

Revista Electrónica Enfermeria Actual en costa Rica

Sivori M., Benzo R., Rhodius E., Jolly E., Boim C., Saadia M., Conti E., Guevara I., Carles D., Victorio C., Santini F., Ratto P., Capparelli I., Prieto E., Azvalinsky M., Alais M., (Julio-Agosto, 2004), Consenso Argentino de Rehabilitación Respiratoria. Sección de rehabilitación respiratoria de la Asociación Argentina de Medicina Respiratoria. 64 (4) $357-367$

Sánchez. J, Díaz. D, Verga. B, Sánchez, L. y Alfonso. P. (Abril/Junio, 2009). Evaluación del Estado Nutricional en Pacientes VIH/SIDA del Municipio San Cristóbal. Revista Ciencias Médicas, 13 (2) 113-122 